\title{
Visual category-driven differences in memory
}

\author{
Adam Steel ${ }^{1}$ and Edward. H. Silson ${ }^{2}$
}

1. Department of Psychology and Brain Sciences, Dartmouth College, Hanover, $\mathrm{NH}$, 03755, USA.

2. Department of Psychology, School of Philosophy, Psychology, and Language Sciences, University of Edinburgh, Edinburgh, UK EH8 9JZ

\begin{abstract}
Categorizing classes of stimuli in the real-world is thought to underlie features of general intelligence, including our ability to infer identities of new objects, environments, and people never encountered before. Our understanding of human categorization, and the neural mechanisms that underlie this ability, was initially described in the context of visual perception. It is now broadly accepted that a network of high-level visual areas on the ventral and lateral surfaces of the brain exhibit some level of 'domain (or category)-selective' activity: preferential neural responses to visual stimuli of one category more than another (e.g., larger responses to faces compared to scenes or manipulable objects). Inspired by this robust and intuitive organization, recent studies have begun investigating the extent to which human memory systems also exhibit a category-selective organization. Surprisingly, this work has revealed strong evidence for the existence of category-selective areas in swaths of cortex previously considered to be domain-general. These results suggest that category-selectivity is a general organizing principle not only of visual cortex, but also for higher-level cortical areas involved in memory. In this chapter we review the evidence for the manifestation of visual category preferences in memory systems, and how this relates to the well-established categoryselectivity exhibited within visual cortex.
\end{abstract}

\section{Emergence of categorical preferences in vision}

Before discussing and evaluating the evidence in favour of category-selective memory systems it is important to describe the well-established category-selective organisation within visual cortex. Neuropsychological, neuroimaging and neurostimulation studies have collectively revealed that human visual cortex contains spatially separate regions that preferentially respond to different categories of stimuli, including (but not restricted to) faces (Fusiform Face Area [FFA], (Kanwisher et al., 1997); Occipital Face Area [OFA], (Silvanto et al., 2010)) bodies (Fusiform Body Area [FBA], (Downing \& Peelen, 2011); Extrastriate Body Area [EBA], (Downing et al., 2001)), objects (Posterior Fusiform [pFs]; Lateral Occipital Cortex [LO], (Malach et al., 1995)), and scenes (Parahippocampal Place Area [PPA] (Epstein and Kanwisher, 1998); Occipital Place Area [OPA] (Hasson et al., 2003; Dilks et al., 2013); Medial Place Area/Retrosplenial Complex [MPA/RSC], (Silson et al., 2016; Epstein, 2008)) (Figure 1A).

These category-selective regions are located across largely distinct swaths of both the lateral and ventral surfaces of the brain. At a macro-scale, these category-selective areas appear to show a repeated organisation, with equivalently selective regions on both surfaces (Figure 1A; Kravitz et al, 2011, 2013, Silson et al., 2015). This duplication does not appear to reflect redundancy or a hierarchical organization. Rather, the areas on the lateral and ventral surfaces likely play distinct roles in human visual perception and cognition, evidenced by their unique functional profiles. For example, areas on the lateral surface (e.g., OPA) exhibit a retinotopic preference for the lower visual field, while areas on the ventral surface (e.g. PPA) exhibit a preference for the upper visual field (Silson et al., 2015).

While both the lateral and ventral occipitotemporal cortex (OTC) have category-selective regions, category-selective activation is most comprehensively studied in ventral OTC, the 
anatomical boundaries of which span the occipitotemporal sulcus (OTS), the collateral sulcus (CoS), and the anterior tip of the mid fusiform sulcus (MFS) (Grill-Spector \& Weiner, 2014; Figure 1B). Moving medially to laterally within this swath of cortex, categorical preference varies systematically from scenes (i.e. PPA) through objects (i.e. pFS), bodies (i.e. FBA) and, finally, faces (i.e FFA) (Figure 1B). Notwithstanding subtle variation in absolute location across individuals, the overall topography of these categorical preferences is remarkably consistent, being present even in infancy (Deen et al., 2017; Kamps et al., 2020) and the congenitally blind (van den Hurk et al., 2017, Mattioni et al., 2020, Murty et al., 2020).

This systematic organization has motivated research into the underlying factors that drive the emergence of category-selectivity, and in particular understanding the relationships between categories. The categorical preferences of both medial and lateral surfaces can be described by two principal axes: A within-category axis (anterior-posterior) and an across-category axis (the medial-lateral axis of ventral OTC) (Figure 1B).

With respect to the within-category axis, it is widely accepted that representations transition from more perceptually oriented to more abstract moving anteriorly (Figure 1B). Consistent with this view, receptive field sizes increase moving anteriorly in OTC, suggesting that these areas play a more integrative role in visual perception (Kravitz et al., 2013), and the response properties of neurons become more holistic and invariant to viewpoint and pose (Rust and DiCarlo, 2010; Eger, Schyns, Kleinschmidt, 2004). This transition towards greater abstraction is also supported by neuroanatomical data, which shows that the anterior portions of the ventral visual stream provide input to the medial temporal lobe memory system, including perirhinal, entorhinal, and hippocampal cortices (Kravitz et al. 2013; Fellman and Van Essen, 1991).

In contrast, multiple theories have been proposed to explain the across-category axis, and thus visual category preference. For example, a prominent theory suggests that categorical preferences occur where they do in the brain because of a pre-existing organisational gradient (i.e. retinotopy) that systematically maps visual field position in the brain (Levy et al. 2001, Wandell et al. 2007). This suggestion comes principally from the observation that scene and face-preferring regions overlap with foveal and peripheral visual field representations, respectively (Levy et al. 2001, Wandell et al. 2007). An alternative account for categorical preferences is that they represent statistical aspects of visual experience, such as real-world size (Konkle and Caramazza, 2015) (e.g. small-large things) or animacy (i.e. animateinanimate) (Mur et al., 2009; Connolly et al. 2012). These theories suggest that categoryselectivity is a derivative of other properties of the visual system. On the other hand, recent work using computational models of the ventral visual stream suggests that the evolution of category-selectivity is a necessary adaptation to accommodate the unique demands associated with discriminating each category (Dobs et al. 2021). Thus, the debate about the functional emergence of visual category preference continues.

Despite continuing debate as to the mechanisms that lead to category-selectivity, its presence within visual cortex is largely accepted. The consistency and intuitiveness of these findings has led to a burgeoning literature investigating the extent to which categorical preferences are present within human memory systems, which we discuss in the following sections. 


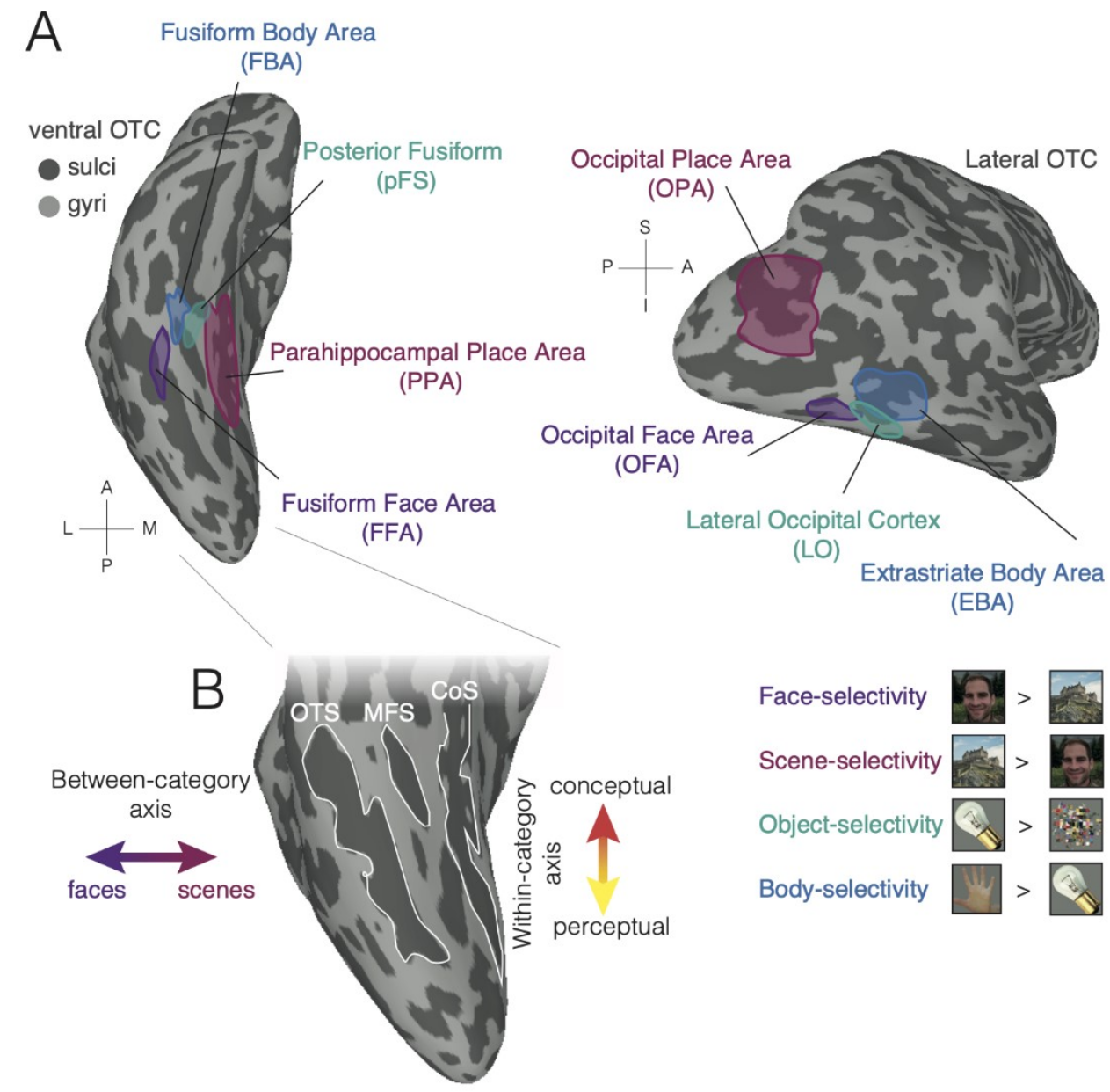

Figure 1: Category-selective regions of visual cortex. A, Category-selective regions are overlaid onto ventral (left) and lateral (right) partially inflated surface reconstructions of the right hemisphere (Faceselective regions $=$ purple, Scene-selective regions $=$ pink, Object-selective regions $=$ green, Bodyselective regions $=$ blue). $A$ repeated organisation can be seen with equivalently selective regions on both surfaces. B, An enlarged version of the ventral surface is depicted with three prominent anatomical sulci highlighting the boundaries of ventral Occipitotemporal cortex (OTC): Occipitotemporal sulcus (OTS), Mid Fusiform Sulcus (MFS) and the Collateral Sulcus (CoS). Ventral OTC is thought to be organised along two axes: A within-category axis, running posterior-anterior and a between-category axis, running medial-lateral.

\section{Expression of categorical preference in memory}

While there is broad agreement that the hippocampus and medial temporal lobe play a vital role in facilitating memory recall (Squire, 1992), there is considerable disagreement regarding the cortical mechanisms that underlie recall. When it comes to understanding the neural substrates underpinning memory recall there exists at least two different perspectives that require careful consideration. The first posits that explicit recall of visual stimuli reinstates perceptual representations and thus recruits the same neural activity patterns that are evoked during visual perception, commonly referred to as "reactivation" or "reinstatement" (Pearson, 2019; McClelland et al., 1995). The second hypothesis questions the reactivation of perceptual representations and instead suggests a perception-memory transition such that memory recall recruits areas adjacent but crucially, anterior of those underpinning perception (Silson et al., 2019; Steel et al., 2021; Favila et al., 2020). In the following sections we explore the evidence for and against both alternatives. 
What evidence exists for the reinstatement framework? Many studies investigating reinstatement have used explicit visual memory recall, i.e. mental imagery (O'Craven \& Kanwisher, 2003). In a typical mental imagery study, participants recall the visual features of a specific stimulus from either long- or short-term memory in response to auditory or visual cues. In a seminal positron emission tomography (PET) imaging study, Kosslyn and colleagues (1994) showed that mental imagery evoked activation in early visual cortex, which had not previously been thought to be active during memory recall. In addition, this activity was topographically organized, such that imagining larger stimuli evoked activity in more peripheral visual areas. Building on this work, subsequent studies using functional magnetic resonance imaging (fMRI) found that the activity of primary visual areas contained stimulus relevant information, including orientation (Albers et al. 2013), shape (Stokes et al, 2009), and visual field position (Slotnick et al, 2005).

This prior work demonstrated that primary visual areas become more active when recalling specific 'low-level' features of a visual stimulus, but do category-selective areas, which are considered 'high-level', also reactivate during mental imagery? Neuroimaging studies provide strong evidence that these regions not only reactivate during mental imagery, but that such reactivation is category-selective in nature (O'Craven and Kanwisher, 2003) and correlates with subjective ratings of vividness (Dijkstra et al. 2017). For example, O'Craven and Kanwisher (2003) investigated the responses of scene-selective PPA and face-selective FFA during both visual perception and mental imagery of scene and face stimuli. Specifically, participants were cued to vividly recall from memory buildings from their college campus (scenes) or the faces of celebrities (faces). As expected, the authors found scene and face preferences in PPA and FFA during visual perception. Remarkably, when they investigated the activity of PPA and FFA during mental imagery, they also observed clear category-specific responses in these regions for their preferred category: PPA and FFA responded robustly during mentally imaged scenes and faces, respectfully, although the magnitude of responses were reduced relative to visual perception (Figure 2A).

Subsequent studies using multivoxel pattern analysis (MPVA) provided additional support for reinstatement by demonstrating that the patterns of activity evoked during the perception of visually presented faces, objects and scenes are recapitulated in ventral OTC during cued retrieval of those same stimuli (Polyn et al., 2005). Despite the sluggishness of the fMRI response relative to the speed of neuronal firing, this work nevertheless showed that these category-selective reactivation patterns during recall were detectable during the recall period and several seconds before the participants verbalised the recalled event - consistent with the idea that category-selective reinstatement in visual cortex is cuing the memory-system to retrieve remembered items (Polyn et al., 2005). Moreover, others have found that mental imagery of scenes and faces can be decoded from PPA and FFA, and in ventral OTC generally, suggesting that the activity in these areas during memory recall carries stimulusspecific visual information (Boccia et al. 2019; Reddy et at., 2010). But are category-selective areas representing fine-grained detail regarding the recalled stimulus, or are they merely representing the category? Evidence suggests the former: mentally imaged object identities can be decoded from object selective pFs (Lee et al., 2012; Sunday et al., 2018) and face identities can be decoded from the FFA (Sunday et al., 2018), although in some cases object identities can also be reliably decoded from face areas (Sunday et al., 2018).

In one very carefully designed experiment (Lee et al., 2012), participants learnt novel names associated with the identity of ten object images (e.g. a 'lekt' for clock, 'dresh' for umbrella). Participants then practiced generating vivid mental images of these same stimuli in a series of three practice phases prior to undergoing an $\mathrm{fMRI}$ scan. During scanning, participants heard the name of the target stimulus (e.g. 'dresh') and were cued to either a perception or imagery trial based on a color change in the fixation cross. The authors compared the responses during 
both perception and imagery in V1 and extrastriate areas (ES) with the responses of objectselective areas LO and pFs. Although object identity could be significantly decoded from all areas during perception, during imagery object decoding was only significant within LO and pFS (Figure 2B).

Finally, in addition to cued recall, fMRI studies suggest that abstract visual properties are maintained in high-level cortex during visual working memory (Christophel et al., 2014). For example, Christophel and colleagues trained a classifier to discriminate complex flow-field patterns from individual participants' fMRI data during perception. Later, when participants were required to hold these same patterns in working memory, the authors were able to decode the direction of flow-field patterns from the activity of motion-selective V5/MT during working memory maintenance, providing support for the importance of region-specific persistent neural activity in working memory. If one considers motion to be a 'category' of stimuli, akin to scenes or faces, then this working memory maintenance is entirely consistent with the evidence discussed above and the reinstatement framework more generally.
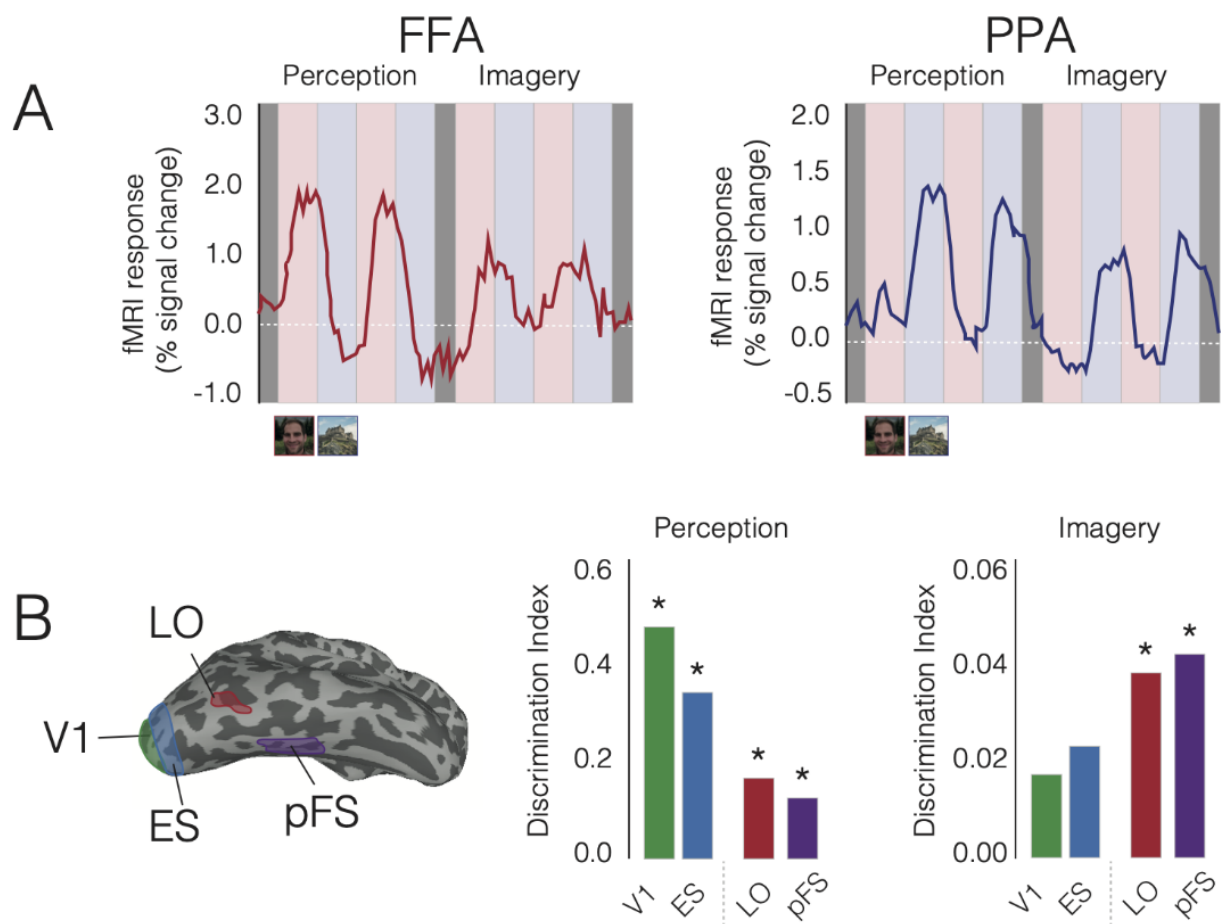

Figure 2: Evidence for the reinstatement framework of memory recall. A, Perceptual and imagery responses from face-selective FFA (left) and scene-selective PPA (right) during face and scene perception and imagery. During perception, both regions exhibit robust activity during the presentation of their preferred category. This categorical-preference is also evident in each region during mental imagery of their preferred category, despite a drop in absolute magnitude. Redrawn with permission from O'Craven \& Kanwisher (2003). B, Perceptual and imagery responses from V1 (green), Extrastriate cortex (blue), object-selective LO (red) and object-selective pFs (purple). Left bars represent object discrimination during perception. Significant object discrimination was present in all regions. Right bars represent object discrimination during imagery. Unlike during perception, significant object discrimination was only evident within object-selective areas $L O$ and $p F s$. Redrawn with permission from Lee et al. (2012). 


\section{Mechanisms of reinstatement activity}

So far, we have discussed evidence in support of the concept that memory recall and working memory maintenance reactivates or reinstates the same neural activity evoked during visual perception. The evidence for reinstatement in early visual areas as well as category-selective regions of high-level visual cortex led some to conclude that the debate about the 'role' of reinstatement in recall was settled (Pearson and Kosslyn, 2015). However, while there is ample evidence that reinstatement does occur, how cortical reinstatement occurs is less clear, although three recent studies provide promising insights into the physiological basis of reinstatement (Breedlove et al. 2020; Favila et al. 2020, Dijkstra et al. 2020).

Collectively, these studies provide empirical evidence that activity during visual memory recall proceeds in a reverse cascade down the visual hierarchy. This activity originates in the medial temporal lobe areas including the hippocampus, and propagates through 'high-level' regions, such as PPA and FFA, to 'low-level' primary visual cortex. Specifically, magnetoencephalography (MEG) decoding analyses suggest that the temporal pattern of activity during visual memory recall matches the activity during perception, but in reverse (Dijkstra et al., 2020). In addition to the reversed temporal pattern, both Breedlove et al (2020) and Favila et al (2020) used fMRI encoding models to show that the computations of the visual hierarchy are reversed, such that the fidelity of information (i.e., spatial blurring) can be predicted by the convolution of the receptive field sizes occurring in reverse. Together, these studies provide strong evidence that domain-general areas, such as the hippocampus, orchestrate the reinstatement of a cortical memory trace, which proceeds down in reverse through the visual hierarchy from high-level to primary sensory areas (Breedlove et al. 2020; Favila et al., 2020; Dijkstra et al., 2020). With regards to category-selectivity, given the intermediate position of category-selective regions in the visual hierarchy, the recruitment of the category-selective visual areas fits naturally within the "reinstatement" framework.

In summary, empirical and theoretical work suggests that category-selective activity evoked during perception appears to be reinstated during memory recall (and working memory). This activity may originate in more traditional memory-systems (e.g., hippocampus). In the next section, we will consider an alternative account, which questions the reinstatement framework and instead argues for an anterior shift between perceptual and mnemonic activity.

\section{Anterior shift of recall-related activity}

The work above provides compelling evidence in favour of the reinstatement framework for both early visual and category-selective areas. But is this the whole story? Neuropsychological patients with lesions in visual cortex suggest that reinstatement alone cannot explain memory recall processes. For example, patients with lesions in visual cortex often report intact mental imagery despite experiencing impaired perception or recognition of specific visual categories (Bertolomeo, 2020; Aguirre \& D'esposito, 1999). This suggests at least a partial anatomical dissociation between the neural substrates of visual perception and memory recall.

How does one reconcile this with the fMRI data discussed above? One potential source of discrepancy is that many experiments investigating category-selective reactivation have investigated this within visually-defined and anatomically constrained regions of interest (ROIs). While these ROI-based analyses are commonplace and highly sensitive to magnitude changes within an area, they necessarily prevent one from observing 1) larger-scale changes that occur outside of the ROI, and 2) changes within subregions of an ROI (for example, shifts in preferences from posterior to anterior). As such, by implicitly presuming that reactivation should occur within a category-selective area, prior work has been largely blind to potential category-selective activity during memory recall outside of these visual cortex ROls. To mitigate this potential confound, recent reports have used resting state functional connectivity (RSFC) to explore new possible ROIs outside of typical visual areas and adopted more holistic data analysis approaches (Baldassano et al., 2013; 2016; Silson et al., 2016). The findings 
from these studies have challenged the purely reactivation account of the memory-recall system by identifying consistent anterior shifts in memory-related activity.

Category-selective activity outside of perceptually-defined cortical areas was initially suggested through investigations of fMRI RSFC patterns (Baldassano et al., 2013; 2016; Silson, Steel et al., 2019). RSFC, which involves examining the correlation between the activity in areas of the brain in the absence of an explicit task, can be used to infer regional communication and establish functional networks (Fox and Raichle, 2007). Based on the suggestion that representations become more abstract anteriorly in the ventral visual system (see within-category axis explanation above), these studies looked for regions of the brain that showed preferential functional connectivity with anterior compared to posterior portions of scene-selective PPA in the hopes of revealing a brain network specialized for conceptual (rather than perceptual) scene understanding. These studies revealed that, as expected, scene-selective areas OPA and RSC/MPA, showed a preferential association with posterior PPA consistent with their role in scene perception; but a series of spatially distinct areas were also shown to preferentially associate with anterior PPA, suggesting that these new areas could play a role in more abstract scene understanding.

Both Silson et al. (2016) and Baldassano et al. (2013) focused on regions associated with anterior PPA in the medial parietal and lateral occipito-parietal cortices. Within medial parietal cortex, a region just anterior of perceptually-driven MPA showed a strong association with anterior PPA, and this more anterior location was consistent with other fMRI work using mental orientation within scenes (Marchette et al., 2014). On the lateral surface of visual cortex, a region in the vicinity of the caudal inferior parietal lobule (cIPL) and anterior of OPA also showed a strong association with anterior PPA. Importantly, the regions exhibiting the stronger association with anterior PPA qualitatively overlapped with regions of cortex shown to be involved during episodic memory recall (Gilmore et al., 2017, 2020) and hinted at the possibility that perception and mental recall recruit distinct, yet adjacent cortical regions.

These initial observations highlighted that perceptual and memory regions of cortex show differential associations within posterior and anterior portions of PPA, respectively. What was unknown at that time, however, was whether or not these anterior regions exhibited categorical preferences. We went on to test this possibility directly using three independent experimental approaches (Silson, Steel et al., 2019). Based on the prior findings that an anterior portion of MPC is strongly associated with anterior PPA, we first tested whether an additional MPC region exhibited a strong association with anterior FFA (Figure $3 \mathbf{A}$ ). Utilizing RSFC, we computed the connectivity between MPC and anterior portions of PPA and FFA, respectively, revealing two spatially distinct regions in MPC (Figure 3B) - a more ventral region (termed MPCv) showed preferential connectivity with anterior PPA and a more dorsal region (termed MPCd) showed preferential connectivity with anterior FFA, suggesting that these regions might exhibit categorical preferences.

This category-specific connectivity was surprising: medial parietal cortex is known to be active during episodic memory-recall (Gilmore et al., 2017; Silson et al., 2019; Gilmore et al., 2019) and future thinking generally, but this region was considered to be at the apex of a domaingeneral processing hierarchy and not thought to contain category-specific subdivisions. To test the categorical preferences of these regions we next analysed the responses to the visual presentation of stimuli spanning six visual categories (Faces, Scenes, Buildings, Bodies, Objects and Scrambled) in an independent group of participants. Unlike category-selective regions of ventral OTC, such as FFA and PPA, which show positively evoked responses during visual perception, both MPC $v$ and MPCd exhibited negative fMRI responses to visually presented stimuli (versus baseline). Crucially, however, and consistent with the connectivity analyses, these negative responses differentiated on the basis of category. That is, responses were least negative (in other words more positive) in MPCV for scenes and least negative in MPCd for faces. The negative overall responses of these regions to visually presented stimuli 
is consistent with this effect in regions of the default mode network (Raichle et al., 2001), but here these negative responses were shown to exhibit categorical preferences.

MPCV and MPCd not only showed strong associations with PPA and FFA, but also exhibited category-selective (albeit negative) responses during visual perception. Coupled with the fact that MPC is involved in memory recall more generally, we went on to test whether these regions would exhibit category-selective memory recall effects in a third independent group of participants. This experiment used an explicit visual memory recall task, where participants were cued to recall personally familiar people (i.e. their mother's face) or places (i.e. their kitchen), intentionally dissociating the spatial and social components of episodic memories. Entirely consistent with the prior results, these areas showed selective activity during mental imagery consistent with their functional connectivity preference (MPCv during place recall, MPCd during people recall) (Figure 3B).

This experiment (Silson, Steel et al., 2019) provided two critical insights. First, it provided direct empirical support for the category-specific memory areas discovered in MPC via functional connectivity. Second, whole-brain analyses that went beyond the initial ROI approach identified four distinct but adjacent regions of MPC that were recruited in a category selective fashion. These areas, which tiled medial parietal cortex along a posterior/ventral to anterior/dorsal arc, showed alternating selectively during memory recall of either specific places or specific people (Figure 3B). Taken together, the results from these three independent experiments provide converging evidence for categorical preferences within distinct regions of MPC. Further, these data suggest a functional link between MPC and category-selective regions of ventral OTC (i.e. PPA, FFA), providing a potential mechanism for the emergence of category-selective memory effects observed. We discuss this possibility in more detail later.

Finally, in a very recent study, we extended the investigation of memory areas specifically in the context of scene perception and recall (Steel et al., 2021). In this work through a series of experiments, we focused on quantifying the difference between perceptual and mnemonic activation for scenes in terms of spatial extent and functional dynamics. First in individual participants, we intensively mapped both the scene-selective perceptual areas using a category-localizer and scene-memory responsive areas using the task from Silson, Steel et al., 2019, which we referred to as the scene-perception and place-memory areas, respectively. We then compared the anatomical locations of these areas. By mapping these regions in individual participants, we were able to quantify the distinction between the perception and memory-related activity in segregation (i.e., is the transition from perception to memory smooth or abrupt) and distance (i.e., how far apart is the peak of perception and memory activation). This experiment revealed a robust anatomical differentiation between the sceneperception and place-memory responsive areas on each cortical surface. The perception and memory areas were highly circumscribed, the place-memory areas were consistently anterior to the scene perception areas, and the separation of the two areas was on the order of centimetres (Figure 3C). Because of their anatomical separation, we referred to these regions as the scene-perception and place-memory areas, respectively.

After demonstrating the anatomical separation between scene-perception and place-memory areas, we went on to assess their potential functional differences. First, we tested whether the place-memory areas responded more strongly to perception of familiar stimuli during a covert recognition task. We found this to be the case: the place-memory areas showed a greater familiarity response than the scene-perception areas. The greater response to familiar compared to unfamiliar images suggests that the place-memory areas are engaged during perception of familiar places. Moreover, this experiment confirmed that the place-memory areas are engaged in bottom-up recognition memory as well as top-down constructive memory recall tasks. 
Finally, in a third experiment, we tested whether the scene-perception and place-memory areas formed distinct neural networks during a naturalistic perception task, which would be further evidence that perception and memory are dissociable neural processes. Participants watched a video comprising architectural tours, college admissions videos, and real-estate advertisements, and we assessed the functional connectivity among the place-memory and scene-perception areas. We found that the scene-perception and place-memory areas have greater within-network compared to between network functional connectivity, suggesting that these areas do form dissociable networks. In addition, consistent with their roles in perception and memory, the scene-perception and place-memory areas were preferentially connected with early visual cortex and the hippocampus, respectively. This study provides strong evidence for category-selective activation in memory, and, moreover, for a partial distinction between the neural substrates of perception and memory in the case of scenes.

A
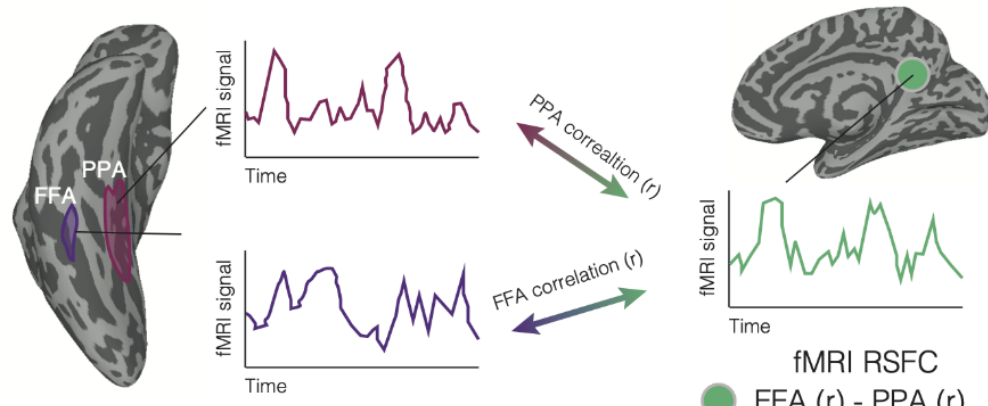

B

Differential RSFC

Memory recall
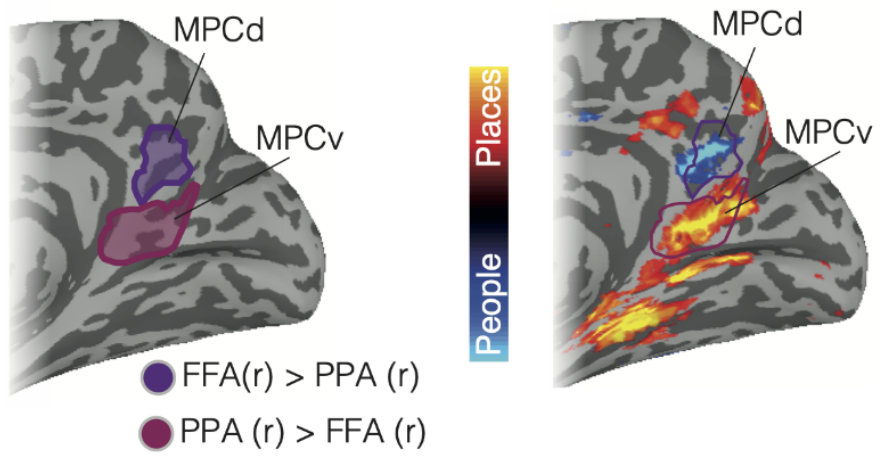

C
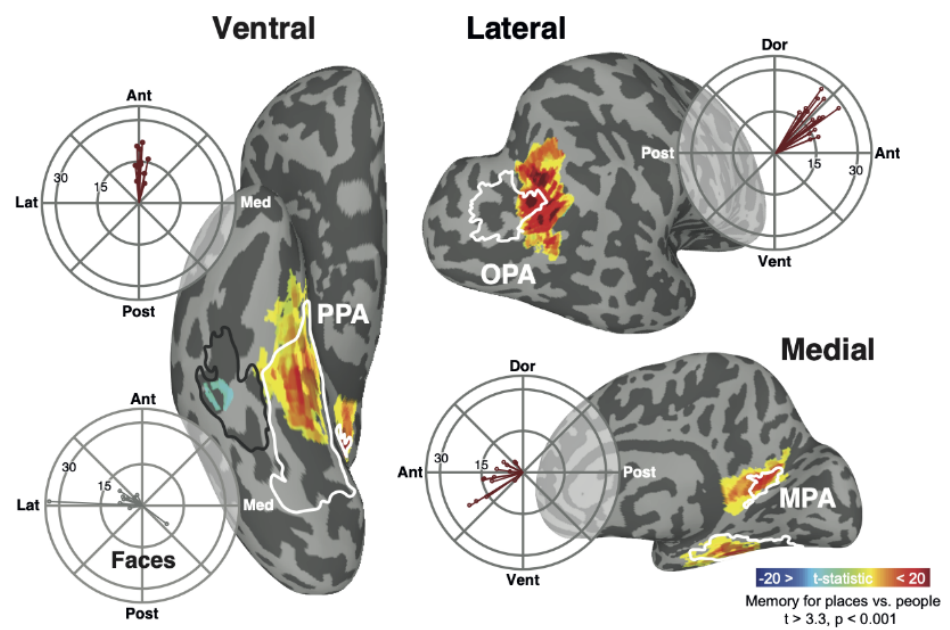

Figure 3: Evidence for an anterior shift in memory recall. A, FFA and PPA are shown on the ventral surface of the right hemisphere. During resting-state functional connectivity analyses, the average response in each region is calculated. For every location in the brain, the correlation in response is 
calculated between FFA and PPA separately, before calculating the difference. B, Left: An enlarged view of medial parietal cortex is shown. Overlaid is the result of the analysis schematised in A. A more ventral region (MPCV) shows preferential connectivity with PPA, whereas a more dorsal region (MPCd) shows preferential connectivity with FFA. Right: Responses during people and place memory recall are overlaid onto the same medial view. Cold-colours represent regions more active during people recall, whereas hot colours represent regions more active during place recall. The ROls defined during the resting-state are overlaid and show a close correspondence. Redrawn with permission from Silson, Steel et al., (2019). C, Distinct topography of place-memory and scene-perception responses in posterior cerebral cortex. The participant's scene perception ROls are outlined in white, and place memory activity is shown in warm colors. The scene-perception network was localized by comparing the BOLD response when participants viewed images of scenes versus with faces (outlined in white, thresholded at vertex-wise $p<0.001)$. Place-memory areas on each surface were localized in separate fMRI runs by comparing the BOLD response when participants recalled personally familiar places versus people (warm colors, thresholded at vertex-wise $p<0.001$ ). Three place-memory areas were observed, each located significantly anterior to one region of the scene-perception network. Polar plots: for each pair of regions, the center of mass of place-memory activation was anterior to the center of mass of scene-perception activation in all participants. In contrast, face memory activation was spatially co-localized with the face-selective fusiform face area (FFA) on the ventral surface, and no anterior shift was observed.

\section{Representational as well as spatial shifts}

The evidence discussed above has focused principally on the relative spatial localisation of perceptual and mnemonic responses, showing a consistent posterior-anterior pattern (Baldassano et al., 2013; 2016; Silson et al., 2019; Steel et al., 2021). Recent work has gone a step further by considering that such posterior-anterior spatial transitions also necessitate content/representational changes (Bainbridge et al. 2020; Favila et al. 2020).

A very recent study (Bainbridge et al., 2020) employed ultra-high resolution 7.0 T fMRI to explore the complexity of representation during perception and recall in high level areas. Bainbridge and colleagues compared activity during encoding and recall of objects and scenes across different levels of conceptual granularity, from coarse (object, scene) through mid (e.g. natural, human-made) to fine (e.g. phone, kitchen). On a given trial, participants were shown a series of stimuli (objects or scenes) and, after a short distraction task, were asked to mentally image one of the stimuli from short-term memory. Consistent with prior work (Silson, Steel et al., 2019), Bainbridge et al. found that recall-related activity was located anterior to perceptual activity in ventral OTC. In addition, the authors investigated the amount of detail (i.e., coarse, mid, and fine levels of granularity) represented in ventral OTC during recall using representational similarity analysis by collapsing across exemplars within each level. Subsequent analyses focused on 1) the similarity of responses during encoding, 2) the similarity of responses during recall and, critically, 3) the similarity of responses between encoding and recall.

These analyses revealed striking differences in the information represented during encoding and recall. During encoding, as expected, detailed stimulus information was present within object- and scene-selective ROls, respectively, even at the fine-grained level. However, during recall, category-selective regions contained very limited detail in their representation: in object selective cortex, only the coarse-level distinction (i.e. object versus scene discrimination) was found to be present and no fine grained distinctions were possible. In the scene-selective areas, this coarse-level distinction was possible in OPA and RSC, and both coarse- and finelevel distinctions of scenes were possible in PPA. Mid-level features were not represented significantly in any area.

Further, within category-selective ROls, very little information was shared between encoding and recall. A cross-decoding analysis revealed only coarse-level information was shared between encoding and recall, and no mid or fine-level information was shared. Finally, the 
authors performed a whole-brain searchlight to see where category-level information could be decoded across the brain. They found that during recall, category-level decoding was only possible in areas anterior to those that contained information during perception. Taken together, these results not only provide convergent evidence for the anterior shift in mnemonic responses, but also suggest that the representational structure of information is distinct between encoding and retrieval.

The change in representational structure and anterior shift in activity highlighted by Silson, Steel et al., (2019), Bainbridge et al. (2020), and Steel et al. (2021) has led to a new theoretical explanation to explain memory reactivation (Favila et al., 2020) that rejects the notion of reactivation. Instead, in their theory, Favila and colleagues introduce the concept of spatial transformations, specifically positing a distinction between perceptual and mnemonic brain areas. The pattern of responses in some brain areas preferentially code perceptual experience, and the pattern of responses in other brain areas preferentially code for those same experiences but when retrieved from memory. In other words, while the memory may have similar content to the encoded event, the spatial distribution of activated areas and the pattern of responses within those areas does not need to be the same.

\section{Origins of visually-driven category-selective activity in memory}

In this Chapter, we have considered two ways in which differences in category-selective memories could occur: either through the reactivation of perceptual regions or via the recruitment of distinct and adjacent regions during recall. But what mechanisms underpin the origins of this category-selective memory activity? Below we outline several related possibilities.

\section{Mirroring of category-selective perceptual activity}

Whether it be for visual analysis or to aid memory recall, overwhelming evidence suggests the brain has found it useful for distinct regions of cortex to exhibit some form of categorical preference, but how do categorically-driven mnemonic preferences develop? One possibility relates to the timeline of maturation between visual cortex and MPC. For instance, recent work suggests that categorical preferences are present in ventral OTC very early on in cortical development (e.g. 6 months: Deen et al., 2017; Kamps et al. 2018) and presumably long before mnemonic mechanisms have been established in MPC (or elsewhere). The adjacency of the category-selective areas observed in MPC revealed by Silson, Steel et al., (2019) is reminiscent of the adjacency of different category-selective regions along the medial-lateral axis of ventral temporal cortex (PPA medial - FFA lateral) suggestive of a category-specific functional link between VTC and MPC. This striking pattern in MPC is highly robust: the people and place preferring regions can be localized reliably in individual participants using both electrophysiology (Woolnough et al. 2020) and fMRI (Steel et al. 2021). Thus, it is possible that the development of categorical-preference in VTC during perception drives the development of categorical-preference in MPC for memory that develops later.

\section{Retinotopic scaffolding in high-level cortex}

A second possibility suggests that retinotopy may be an organizational scaffold outside the visual system, including in high-level cortical regions like medial and lateral parietal cortex. This is related to the influential theory regarding the development of categorical preferences in visual cortex, which posits that visual category-selectivity may be constrained by retinotopy that is set-up in-utero via retinal waves (Wong 1999; Ackman et al., 2012), that is, prior to visual experience. This data highlights that retinotopic biases are not only present prior to the formation of category-selectivity (Arcaro and Livingstone, 2017), but that they persist even if the formation of category-selectivity is prevented through deprivation (Arcaro and Livingstone, 2019). Such a retinotopic explanation for the emergence of categorical preferences in visual cortex could also apply to the formation of category-selective memory areas, in particular 
within MPC. Indeed, exploratory analyses using resting-state functional connectivity (Silson et al., 2019) highlights that people and place memory areas of MPC show differentially strong connections with foveal and peripheral portions of early visual cortex and may have retinotopic-like response properties (Szinte \& Knapen, 2019). It is possible that the retinotopic preferences of regions within early visual cortex and beyond (e.g. FFA foveally biased, PPA peripherally biased) are utilized during the emergence of categorical preferences in memory recall, but further, more targeted research is required to unpack this.

\section{Alternative explanations to category-selectivity in high-level cortex}

Finally, perhaps the apparent category-selectivity in high-level cortex is a red herring, and such responses are a by-product of the cognitive functions implemented in these regions. In this view, category-selectivity is not a feature of high-level cortex at all. Instead, the appearance of category-selectivity is due to activation of large-scale cortical systems that are specialized for specific computations, rather than operate on visual categories, per se. One leading theory emphasizes differential networks specialized for making inferences based on episodic memory projection or theory of mind (Braga et al., 2017, 2019; DiNicola et al., 2020). Below we provide a brief description of this theory.

Rather than higher-level cortex instantiating the precise details of place and people related information directly, Braga and colleagues propose that two interdigitated networks are separately involved in episodic memory projection and theory of mind computations, respectively. This theory is laid out in a series of papers from this group. First, using restingstate functional connectivity in individual participants, Braga and Buckner $(2017 ; 2019)$ discovered two interdigitated networks that extended throughout high-level cortex. Notably, although these networks broadly spanned cortex, the posterior aspects of these areas overlap extensively with the face- and scene- recall-related areas reported on the medial surface in Silson, Steel et al., (2019), and with the place-memory network areas reported in Steel et al. (2021). After identifying these parallel networks, Braga's team, compared the activity of these networks using two different tasks, an episodic projection task and a theory of mind task (DiNicola et al., 2020). In the episodic projection task, participants either imagined or recalled hypothetical situations related to themselves. In contrast, in the theory-of-mind task, participants read stories about characters that held false beliefs or experienced physical or emotional pain (DiNicola et al. 2020). Consistent with the overlap between these networks and the face- and scene- recall-related activity reported by Silson, Steel et al. (2019b), DiNicola and colleagues found a dissociation between the network activity in response to these two tasks. They suggest that these large scale networks serve to instantiate episodic projection and theory of mind computations, and propose therefore that category-selective activity is an epiphenomenon related to the overall function of these regions.

In addition to the studies by Braga's team, others have found similar functional differentiation for social and spatial judgements in medial and lateral parietal cortex. For example, distinct areas of parietal cortex are activated when participants make judgments of social, spatial, and temporal distances (e.g. "which of two individuals is closer to you?", "which of these places are you closer to," and "which of two events happened closer to the present day?", (Peer et al., 2015)). Interestingly, not only do these areas activate during these distance judgments, but also the activity of these areas appear to represent physical distances in the environment, suggesting that they play a causal role in these types of inferences. For example, the activity evoked during spatial judgments in ventral MPC appears to be mapped according to physical scale (i.e. Euclidean distance; Peer et al. 2019; Morton et al., 2021). In contrast, the activity of the dorsal MPC cortex appears to map social network distance (i.e., associative distance; Parkinson et al., 2018; Peer et al. 2020). This has led to the suggestion that these areas are specifically involved in distance estimation and serve as key nodes for inferences that utilize these metrics (Peer et al., 2020). Importantly, these modality-specific distance representations 
are in anatomically similar locations to the areas involved in face- and scene- recall and overlap with the networks proposed by Braga and colleagues.

How do these alternative frameworks from Braga and colleagues and Peer and colleagues explain category-selectivity in high-level cortex? Their theories suggest that explicit recall of faces would necessarily recruit the theory-of-mind/associative distance networks because participants are recalling information about people. Likewise, the episodic memory projection/Euclidean distance network is necessarily recruited when participants recall personally familiar places because of this network's role in scene construction. Therefore, rather than being related to the visual category domains, these networks perform specific functions for cognitive inference. Future work might consider means of dissociating the visualcategory information from the inference conditions to distinguish between these theories, for example, investigating whether the theory-of-mind network is active for social inference tasks where actors are not people, such as animals.

\section{Future directions and outstanding questions}

Category-selectivity is a robust phenomenon observable throughout the brain. Here, we reviewed recent evidence suggesting that category-selective visual areas also activate in a selective manner during memory-recall. Moreover, category-selective memory areas may exist outside of the classically defined visual areas, suggesting that category-selectivity may be a fundamental organizing property of the brain. These discoveries open many outstanding questions to be addressed by future studies.

- Do category-selective areas exist in high-level cortex for the recall of visual categories besides scenes and faces (e.g., objects, bodies, etc)?

- What functional properties differentiate the visual and memory category-selective areas?

- When do the category-selective memory areas develop relative to visual areas?

- Are category-selective regions present in other domain-general areas of the brain, such as medial and lateral prefrontal cortex?

- How are recalled memories represented within category-selective regions of visual and medial parietal cortex?

\section{Summary and conclusion}

In summary, recent evidence suggests that category-selective activity occurs throughout the brain during memory recall. This activity is both within category-selective visual areas, as well as in areas of the brain classically considered to be domain-general. Although reactivation of perceptual processes may occur during visual memory recall, such 'reactivation' is likely not sufficient to explain memory recall mechanisms. Indeed, the recent and consistent evidence for an 'anterior shift' in locus of activity during memory recall (Silson, Steel et al., 2019; Bainbridge et al., 2020; Favila et al., 2020) strongly challenges the reactivation framework and suggests the brain undertakes spatial and potentially representational transformations during visual memory recall. Notwithstanding debate around the mechanism for visual memory recall, the evidence suggests that categorical preferences are not only a property of visual cortex regions, but that such preferences extend into the brain's memory systems as well. Understanding the origins of such visually-driven categorical differences in memory is a key goal of the future.

\section{References}

Ackman, J. B., Burbridge, T. J., \& Crair, M. C. (2012). Retinal waves coordinate patterned activity throughout the developing visual system. Nature, 490(7419), 219-225. 
Arcaro, M. J., \& Livingstone, M. S. (2017). A hierarchical, retinotopic proto-organization of the primate visual system at birth. Elife, 6, e26196.

Aguirre, G. K., \& D'Esposito, M. (1999). Topographical disorientation: a synthesis and taxonomy. Brain, 122(9), 1613-1628.

Albers, A. M., Kok, P., Toni, I., Dijkerman, H. C., \& De Lange, F. P. (2013). Shared representations for working memory and mental imagery in early visual cortex. Current Biology, 23(15), 1427-1431.

Baldassano, C., Beck, D. M., \& Fei-Fei, L. (2013). Differential connectivity within the parahippocampal place area. Neuroimage, 75, 228-237.

Baldassano, C., Esteva, A., Fei-Fei, L., \& Beck, D. M. (2016). Two distinct scene-processing networks connecting vision and memory. Eneuro, 3(5).

Bainbridge, W. A., Hall, E. H., \& Baker, C. I. (2020). Distinct representational structure and localization for visual encoding and recall during visual imagery. Cerebral Cortex, bhaa329.

Bartolomeo, P., Hajhajate, D., Liu, J., \& Spagna, A. (2020). Assessing the causal role of early visual areas in visual mental imagery. Nature Reviews Neuroscience, 21(9), 517-517.

Boccia, M., Bonavita, A., Diana, S., Di Vita, A., Ciurli, M. P., \& Guariglia, C. (2019). Topographical disorientation: clinical and theoretical significance of long-lasting improvements following imagerybased training. Frontiers in human neuroscience, 13, 322.

Braga, R. M., \& Buckner, R. L. (2017). Parallel interdigitated distributed networks within the individual estimated by intrinsic functional connectivity. Neuron, 95(2), 457-471.

Braga, R. M., Van Dijk, K. R., Polimeni, J. R., Eldaief, M. C., \& Buckner, R. L. (2019). Parallel distributed networks resolved at high resolution reveal close juxtaposition of distinct regions. Journal of neurophysiology, 121(4), 1513-1534.

Breedlove, J. L., St-Yves, G., Olman, C. A., \& Naselaris, T. (2020). Generative feedback explains distinct brain activity codes for seen and mental images. Current Biology, 30(12), 2211-2224.

Connolly, A. C., Guntupalli, J. S., Gors, J., Hanke, M., Halchenko, Y. O., Wu, Y. C., ... \& Haxby, J. V. (2012). The representation of biological classes in the human brain. Journal of Neuroscience, 32(8), 2608-2618.

Christophel, T. B., \& Haynes, J. D. (2014). Decoding complex flow-field patterns in visual working memory. Neuroimage, 91, 43-51.

Deen, B., Richardson, H., Dilks, D. D., Takahashi, A., Keil, B., Wald, L. L., \& Saxe, R. (2017). Organization of high-level visual cortex in human infants. Nature communications, 8(1), 1-10.

Downing, P. E., \& Peelen, M. V. (2011). The role of occipitotemporal body-selective regions in person perception. Cognitive neuroscience, 2(3-4), 186-203.

Downing, P. E., Jiang, Y., Shuman, M., \& Kanwisher, N. (2001). A cortical area selective for visual processing of the human body. Science, 293(5539), 2470-2473.

Dilks, D. D., Julian, J. B., Paunov, A. M., \& Kanwisher, N. (2013). The occipital place area is causally and selectively involved in scene perception. Journal of Neuroscience, 33(4), 1331-1336.

Dijkstra, N., Ambrogioni, L., Vidaurre, D., \& van Gerven, M. (2020). Neural dynamics of perceptual inference and its reversal during imagery. Elife, 9, e53588.

Dijkstra, N., Zeidman, P., Ondobaka, S., van Gerven, M. A., \& Friston, K. (2017). Distinct top-down and bottom-up brain connectivity during visual perception and imagery. Scientific reports, 7(1), 1-9. 
DiNicola, L. M., Braga, R. M., \& Buckner, R. L. (2020). Parallel distributed networks dissociate episodic and social functions within the individual. Journal of neurophysiology, 123(3), 1144-1179.

Dobs, K., Martinez, J., Kell, A.J., \& Kanwisher, N. (2021). Brain-like functional specialization emerges spontaneously in deep neural networks. bioRxiv, 2021.07.05.451192.

Eger, E., Schyns, P. G., \& Kleinschmidt, A. (2004). Scale invariant adaptation in fusiform faceresponsive regions. Neuroimage, 22(1), 232-242.

Epstein, R., \& Kanwisher, N. (1998). A cortical representation of the local visual environment. Nature, 392(6676), 598-601.

Epstein, R. A. (2008). Parahippocampal and retrosplenial contributions to human spatial navigation. Trends in cognitive sciences, 12(10), 388-396.

Favila, S. E., Lee, H., \& Kuhl, B. A. (2020). Transforming the Concept of Memory Reactivation. Trends in neurosciences.

Favila, S. E., Kuhl, B. A., \& Winawer, J. (2020). Perception and memory have distinct spatial tuning properties in human visual cortex. bioRxiv, 811331 .

Felleman, D. J., \& Van Essen, D. C. (1991). Distributed hierarchical processing in the primate cerebral cortex. Cerebral cortex (New York, NY: 1991), 1(1), 1-47.

Fox, M. D., \& Raichle, M. E. (2007). Spontaneous fluctuations in brain activity observed with functional magnetic resonance imaging. Nature reviews neuroscience, 8(9), 700-711.

Gilmore, A. W., Nelson, S. M., \& McDermott, K. B. (2015). A parietal memory network revealed by multiple MRI methods. Trends in cognitive sciences, 19(9), 534-543.

Gilmore, A. W., Quach, A., Kalinowski, S. E., Gotts, S. J., Schacter, D. L., \& Martin, A. (2021). Dynamic content reactivation supports naturalistic autobiographical recall in humans. Journal of Neuroscience, 41(1), 153-166.

Grill-Spector, K., \& Weiner, K. S. (2014). The functional architecture of the ventral temporal cortex and its role in categorization. Nature Reviews Neuroscience, 15(8), 536-548.

Kamps, F. S., Hendrix, C. L., Brennan, P. A., \& Dilks, D. D. (2020). Connectivity at the origins of domain specificity in the cortical face and place networks. Proceedings of the National Academy of Sciences, 117(11), 6163-6169.

Kanwisher, N., McDermott, J., \& Chun, M. M. (1997). The fusiform face area: a module in human extrastriate cortex specialized for face perception. Journal of neuroscience, 17(11), 4302-4311.

Kravitz, D. J., Peng, C. S., \& Baker, C. I. (2011). Real-world scene representations in high-level visual cortex: it's the spaces more than the places. Journal of Neuroscience, 31(20), 7322-7333.

Kravitz, D. J., Saleem, K. S., Baker, C. I., Ungerleider, L. G., \& Mishkin, M. (2013). The ventral visual pathway: an expanded neural framework for the processing of object quality. Trends in cognitive sciences, 17(1), 26-49.

Konkle, T., Wang, X., Peelen, M., Caramazza, A., \& Bi, Y. (2015). Convergence and divergence in the neural organization of object responses to pictures and words. Journal of Vision, 15(12), 375-375.

Kosslyn, S. M., \& Ochsner, K. N. (1994). In search of occipital activation during visual mental imagery. Trends in neurosciences, 17(7), 290-292.

Stokes, M. G., Atherton, K., Patai, E. Z., \& Nobre, A. C. (2012). Long-term memory prepares neural activity for perception. Proceedings of the National Academy of Sciences, 109(6), E360-E367. 
Lee, S. H., Kravitz, D. J., \& Baker, C. I. (2012). Disentangling visual imagery and perception of realworld objects. Neuroimage, 59(4), 4064-4073.

Levy, I., Hasson, U., Avidan, G., Hendler, T., \& Malach, R. (2001). Center-periphery organization of human object areas. Nature neuroscience, 4(5), 533-539.

Malach, R., Reppas, J. B., Benson, R. R., Kwong, K. K., Jiang, H., Kennedy, W. A., \& Tootell, R. B. (1995). Object-related activity revealed by functional magnetic resonance imaging in human occipital cortex. Proceedings of the National Academy of Sciences, 92(18), 8135-8139.

Marchette, S. A., Vass, L. K., Ryan, J., \& Epstein, R. A. (2014). Anchoring the neural compass: coding of local spatial reference frames in human medial parietal lobe. Nature neuroscience, 17(11), 1598.

Mattioni, S., Rezk, M., Battal, C., Bottini, R., Mendoza, K. E. C., Oosterhof, N. N., \& Collignon, O. (2020). Categorical representation from sound and sight in the ventral occipito-temporal cortex of sighted and blind. Elife, 9, e50732.

McClelland, J. L., McNaughton, B. L., \& O'Reilly, R. C. (1995). Why there are complementary learning systems in the hippocampus and neocortex: insights from the successes and failures of connectionist models of learning and memory. Psychological review, 102(3), 419.

Morton, N. W., Zippi, E. L., Noh, S., \& Preston, A. R. (2021). Semantic knowledge of famous people and places is represented in hippocampus and distinct cortical networks. Journal of Neuroscience.

Mur, M., Bandettini, P. A., \& Kriegeskorte, N. (2009). Revealing representational content with patterninformation fMRI —an introductory guide. Social cognitive and affective neuroscience, 4(1), 101-109.

Murty, N. A. R., Teng, S., Beeler, D., Mynick, A., Oliva, A., \& Kanwisher, N. (2020). Visual experience is not necessary for the development of face-selectivity in the lateral fusiform gyrus. Proceedings of the National Academy of Sciences, 117(37), 23011-23020.

O'Craven, K. M., \& Kanwisher, N. (2000). Mental imagery of faces and places activates corresponding stimulus-specific brain regions. Journal of cognitive neuroscience, 12(6), 1013-1023.

Parkinson, C., Kleinbaum, A. M., \& Wheatley, T. (2018). Similar neural responses predict friendship. Nature communications, 9(1), 1-14.

Pearson, J. (2019). The human imagination: the cognitive neuroscience of visual mental imagery. Nature Reviews Neuroscience, 20(10), 624-634.

Pearson, J., \& Kosslyn, S. M. (2015). The heterogeneity of mental representation: Ending the imagery debate. Proceedings of the National Academy of Sciences, 112(33), 10089-10092.

Peer, M., Hayman, M., Tamir, B., \& Arzy, S. (2019). Brain coding of social network structure. bioRxiv, 850065.

Peer, M., Ron, Y., Monsa, R., \& Arzy, S. (2019). Processing of different spatial scales in the human brain. Elife, 8, e47492.

Peer, M., Salomon, R., Goldberg, I., Blanke, O., \& Arzy, S. (2015). Brain system for mental orientation in space, time, and person. Proceedings of the National Academy of Sciences, 112(35), 1107211077.

Polyn, S. M., Natu, V. S., Cohen, J. D., \& Norman, K. A. (2005). Category-specific cortical activity precedes retrieval during memory search. Science, 310(5756), 1963-1966.

Reddy, L., Tsuchiya, N., \& Serre, T. (2010). Reading the mind's eye: decoding category information during mental imagery. Neuroimage, 50(2), 818-825. 
Rust, N. C., \& DiCarlo, J. J. (2010). Selectivity and tolerance ("invariance") both increase as visual information propagates from cortical area V4 to IT. Journal of Neuroscience, 30(39), 12978-12995.

Silson, E. H., Chan, A. W. Y., Reynolds, R. C., Kravitz, D. J., \& Baker, C. I. (2015). A retinotopic basis for the division of high-level scene processing between lateral and ventral human occipitotemporal cortex. Journal of Neuroscience, 35(34), 11921-11935.

Silson, E. H., Gilmore, A. W., Kalinowski, S. E., Steel, A., Kidder, A., Martin, A., \& Baker, C. I. (2019). A posterior-anterior distinction between scene perception and scene construction in human medial parietal cortex. Journal of Neuroscience, 39(4), 705-717.

Silson, E. H., Steel, A., Kidder, A., Gilmore, A. W., \& Baker, C. I. (2019). Distinct subdivisions of human medial parietal cortex support recollection of people and places. Elife, 8, e47391.

Silson, E. H., Steel, A. D., \& Baker, C. I. (2016). Scene-selectivity and retinotopy in medial parietal cortex. Frontiers in human neuroscience, 10, 412.

Silvanto, J., Schwarzkopf, D. S., Gilaie-Dotan, S., \& Rees, G. (2010). Differing causal roles for lateral occipital cortex and occipital face area in invariant shape recognition. European Journal of Neuroscience, 32(1), 165-171.

Slotnick, S. D., Thompson, W. L., \& Kosslyn, S. M. (2005). Visual mental imagery induces retinotopically organized activation of early visual areas. Cerebral cortex, 15(10), 1570-1583.

Steel, A., Billings, M. M., Silson, E. H., \& Robertson, C. E. (2021). A network linking scene perception and spatial memory systems in posterior cerebral cortex. Nature communications, 12(1), 1-13.

Sunday, M. A., McGugin, R. W., Tamber-Rosenau, B. J., \& Gauthier, I. (2018). Visual imagery of faces and cars in face-selective visual areas. PloS one, 13(9), e0205041.

Squire, L. R. (1992). Memory and the hippocampus: a synthesis from findings with rats, monkeys, and humans. Psychological review, 99(2), 195.

Szinte, M., van Es, D. M., \& Knapen, T. (2019). The visual selectivity of the default mode network. Journal of Vision, 19(10), 212-212.

van den Hurk, J., Van Baelen, M., \& de Beeck, H. P. O. (2017). Development of visual category selectivity in ventral visual cortex does not require visual experience. Proceedings of the National Academy of Sciences, 114(22), E4501-E4510.

Wandell, B. A., Dumoulin, S. O., \& Brewer, A. A. (2007). Visual field maps in human cortex. Neuron, 56(2), 366-383.

Woolnough, O., Rollo, P. S., Forseth, K. J., Kadipasaoglu, C. M., Ekstrom, A. D., \& Tandon, N. (2020). Category Selectivity for Face and Scene Recognition in Human Medial Parietal Cortex. Current Biology, 30(14), 2707-2715.

Wong, R. O. (1999). Retinal waves and visual system development. Annual review of neuroscience, 22(1), 29-47. 